\title{
Prozesse der Entscheidungsfindung - geographische Zugänge und Perspektiven. Einführung zum Themenheft
}

\section{Joris Van Wezemael, Freiburg, Marc Zaugg Stern, Zürich}

\begin{abstract}
« Thanks for Nothing Fema», was the message to the national emergency agency on one dislodged building; a simple «Baghdad was emblazoned on another. Debris was everywhere» (THE GUARDIAN vom 29.08.2007 über die Situation nach Hurrikan Katrina in New Orleans).
\end{abstract}

Das Iraq Body Count Project (IBC), das der Dokumentation des 2003 von der Administration Bush begonnenen Irak-Kriegs verpflichtet ist, zählt für den 23. August 2005 mindestens 19 Kriegsopfer (Iraq Body Count Project, IBC, 2008). An demselben 23. August 2005 bildete sich über den Bahamas der Hurrikan Katrina, der am 29. August auf New Orleans treffen sollte. Katrina kostete mindestens 1'800 Menschen das Leben. Die Schäden wurden in einem Bericht vom US-amerikanischen National Climatic Data Center (NCDC 2005) auf 126 Milliarden Dollar geschätzt. Der gleiche Bericht geht davon aus, dass mindestens 250'000 Menschen vor Katrina und dessen direkten Folgen wie Überschwemmungen oder Erdrutschen fliehen mussten. Die Bevölkerung von New Orleans verringerte sich wegen Katrina zeitweise von 490'000 auf 250'000 Menschen.

Obschon die beiden Ereignisse vom 23. August 2005 im Irak und über den Bahamas tausende Kilometer voneinander entfernt und in ihren Ursachen unabhängig erscheinen, ist das Eine ohne das Andere in dessen Konsequenzen nicht verstehbar. Die Wahrnehmungen der Ereignisse, deren Diskurse und die auf sie bezogenen Massnahmen bedingen sich gegenseitig. Vermutlich hätte eine politische Diskussion in der ersten Hälfte des 20. Jahrhunderts diese beiden Ereignisse nicht miteinander verknüpft. In der Anfang des 21. Jahrhunderts vor dem Hintergrund sich globalisierender Öffentlichkeiten stattfindenden Debatte über Ursachen und Folgen von Katrina wurden die beiden Themen jedoch schnell diskursiv miteinander und ineinander verwoben: zum Einen wurden die finanziellen Mittel, die in den IrakKrieg investiert wurden, thematisiert. Gerade in den Medien wurde die Kritik heftig und nicht immer sachlich geführt, so war gar von einer schlecht organisierten, inkompetent geführten und überfordert agierenden Bundesbehörde Federal Emergency Management Agency (FEMA) die Rede, der zudem auch die Mittel für die Katastrophenprävention und -bewältigung in den USA gefehlt hätten. Zum Anderen wurde der Mangel an personellen Ressourcen bei der Bewältigung von Katrina ebenfalls mit dem Irak-Krieg in Zusam- menhang gebracht: die dort eingesetzten Reservisten limitierten die Einsatzkräfte, die den Verantwortlichen des Bundes und der betroffenen Bundesstaaten zur Verfügung standen. In der heftigen Diskussion über Katrina vermischten sich der Irak-Krieg und die Überforderung der Bundesbehörden mit weiteren Themen wie etwa der fehlenden Gefahrenvorsorge in New Orleans oder dem «racial issue»-der besonderen Verletzlichkeit insbesondere afro-amerikanischer Bevölkerungsgruppen.

Aus einer geographisch-disziplinären Perspektive verknüpfte die Debatte über Katrina nicht nur sehr unterschiedliche Themenfelder miteinander, sondern unterminierte auch die traditionellerweise in der Geographie proklamierten hierarchischen Skalenzusammenhänge bei der Auseinandersetzung mit einem politischen Gegenstand (lokal - regional - national global) und die damit zusammenhängende Frage nach der richtigen Ebene für die Entscheidungsfindung. Angesichts der Komplexität heutiger Entscheidungsfindung ist deshalb eine Diskussion über Annahmen, Zugänge und Ergebnisse der geographischen Auseinandersetzung mit Prozessen der Entscheidungsfindung notwendig. Letzteres war 2007 Anlass zur Gründung des Arbeitskreises Geographie und Prozesse der Entscheidungsfindung (AK GPE) (VAN WEZEMAEL \& Zaugg Stern 2006). Die Durchführung der konstitutiven Sitzung am Deutschen Geographentag 2007 in Bayreuth mündete in der Publikation des vorliegenden Geographica Helvetica-Themenheftes.

Ausgangspunkt des Themenheftes bildet die These, dass aus der Vielschichtigkeit heutiger Problemstellungen laufend neue Formen der Entscheidungsfindung entstehen. Diese können bestehende Verfahren ergänzen oder auch unterminieren. Im Rahmen dieser «alternativen» Formen der Entscheidungsfindung verändert sich erstens die Beziehung von «Laien» und «Experten» (vgl. den Beitrag von SABINE HAFNER). Zweitens wird der Blick auf die bei komplexen Entscheidungsfindungsprozessen ersichtlichen multiplen Rationalitäten frei (vgl.den Beitrag von Oliver IBERT): Ausgehend von multiplen Wahrheiten und Wirklichkeiten respektive verschiedenen Reichweiten «wahrer» Aussagen werden prozedurale Festlegungen im Rahmen des Entscheidungsfindungsprozesses aufgewertet zulasten des im Rahmen «moderner» Entscheidungsfindung als vorrangig proklamierten Expertenwissens. Der Beitrag von Joris VAN Wezemael \& MatTHIAs Loepfe weist drittens darauf hin, dass infolge der Komplexität heutiger Problemstellungen häufig auch Entscheidungen 
vor dem Hintergrund nicht auszuräumender Unsicherheit(-en) getroffen werden (müssen). Viertens zeigen die Beiträge von HAFNER, IBERT, TrufFer \& StÖRMER sowie Van Wezemael \& Loepfe, dass die Aufwertung von Fragen der Legitimität bei der Entscheidungsfindung dazu führt, dass fortbestehende Ziel- und Interessendivergenzen keinen Widerspruch zu einer mehr oder minder erfolgreichen Lösungsfindung darstellen müssen (z.B. partizipative, konsensuale, kollek tive oder «Governanz»-orientierte Formen der Entscheidungsfindung). Fünftens zeigt der Beitrag von BERnHaRD TRUFFER \& ECKHARD STÖRMER, dass alternative Verfahren auch strategisch dazu eingesetzt werden können, um die «Blackbox» tradierter Verfahren aufzubrechen und somit bislang nicht bedachte Möglichkeitsfelder zu erschliessen. Sechstens weisen alternative Verfahren der Entscheidungsfindung auf ein Paradoxon hin: Sie setzen bestehende und funktionierende Institutionen zur Meinungsbildung und Entscheidungsfindung (liberaldemokratische Verfahren) voraus und untergraben diese zugleich (vgl. hierzu den von BenEDIKT KoRf verwendeten Begriff des «Ausnahmezustands»).

\section{Zielsetzung des Themenheftes}

Ausgangspunkt dieses Themenheftes bildet die Zielsetzung, Entscheidungsfindungen als geographisches Forschungsobjekt zu explizieren und fachbereichsübergreifend zu thematisieren. Daher bilden für einmal nicht geographische Regionen, Diskurse oder Sub-Disziplinen den gemeinsamen Nenner der Beiträge. Die Explizierung von Entscheidungsfindung in den verschiedenen Bereichen lässt die Beiträge miteinander in Beziehung treten, wodurch fachhistorische Grenzen fruchtbringend überschritten werden. Über die Auseinandersetzung mit Entscheidungsfindungsprozessen werden diese verschiedenen, regional und/oder thematisch verorteten wissenschaftlichen Gemeinschaften oder Diskurse zusammengeführt. Hiermit soll eine breite Fachdebatte über die Rolle der Geographie an der Schnittstelle von Wissenschaft(en), Politik, Ökonomie und Gesellschaft eröffnet werden. Freilich wäre es anmassend, in einem einzigen Heft das ganze Potential dieser Herangehensweise einlösen zu wollen. Die folgenden Beiträge nehmen vornehmlich eine Verknüpfung von wirtschafts- und sozialgeographischen Debatten über individuelle und kollektive Entscheidungsfindung vor. So treffen Beiträge aus der Politischen Geographie auf solche aus der regionalen Innovationsforschung oder den Regional and Urban Studies.

Ein Set von Fragen eröffnet einen diskursiven Raum. Diese thematisieren Grundannahmen geographischer Arbeiten zur Entscheidungsfindung und die Bedeutung, welche die Annahme gesteigerter Komplexität aktueller Entscheidungsfindungsprozesse für die geographische Forschung zeitigt. Wie spiegeln sich theoretische Postulate oder empirisch ersichtliche Strategien zur adäquaten Komplexitätsreduktion in den wissenschaftlichen Konzepten? Die Analyse von Entscheidungsfindung fragt nach den Prinzipien zur Abgrenzung von Prozessen oder Systemen, in deren Rahmen Entscheidungen vorbereitet und gefällt werden. Die den Beiträgen zugrunde liegende Annahme gesteigerter Komplexität stellt epistemologische Konzepte (z.B. Akteurmodelle) in Frage und ruft nach ontologischen Fundierungen zur Untersuchung und Konzeptualisierung von Entscheidungsfindungsprozessen. Schliesslich steht das Zusammenspiel der unterschiedlichen system- und lebensweltlich fundierten Rationalitäten zur Diskussion.

Die Autoren und Autorinnen in diesem Themenheft widmen sich diesen Fragen aus unterschiedlichen Perspektiven. Sie tragen durch die Reflexion in ihren Beiträgen Bausteine zu einem neuen, der Komplexität heutiger Entscheidungsfindung angemessenen Verständnis bei. Zugleich zeigen sie auf, dass unterschiedliche Perspektiven zum Thema notwendig sind, diese jedoch auch jeweils eigenständige und sich voneinander unterscheidende Erkenntnisspuren legen.

ECKHARD STÖRMER und BERNHARD TRUFFER fokussieren in ihrer action research den Prozess der Entscheidungsfindung und thematisieren hierbei sowohl den Akteurbegriff (soziotechnische Entitäten) als auch Governanz-Situationen. Sie thematisieren in ihrem Beitrag die lock-in-Situation in Entscheidungsfindungen bezüglich langlebiger Infrastruktursysteme im Abwasserbereich. Ihres Erachtens bedürfen diese neuer Methoden im Umgang mit der Unsicherheit über zukünftige Entwicklungen der Rahmenbedingungen (z.B. Siedlungsentwicklung, Wirtschaftsentwicklung, Regulationsweisen) und Potentialen des Systems. Mit «Regional Infrastructure Foresight»-Methoden (RIF-Methoden) kann der Unsicherheit begegnet werden, indem verschiedene denkbare Zukünfte vorgestellt werden. Der Beitrag zeigt auf, dass Entscheidungsergebnisse Lösungen sind, die den vielfältigen Kriterien verschiedener Zukunftswege gerecht werden müssen, um robust für die Lebensdauer der soziotechnischen Entitäten zu sein.

SABINE HAfNers Interesse gilt in erster Linie dem Resultat eines partizipativen Verfahrens im Bereich der Quartierentwicklung. Hierbei weist sie auf den Spannungsbogen zwischen deliberativem Potential und instrumentellem Einsatz von partizipativen Verfahren hin. Sie argumentiert, dass in der Stadtentwicklungspolitik gesellschaftliche Umbruchprozesse kumulieren. Hafner illustriert dies am Beispiel des Programms «Soziale Stadt», welches sie als «kollektiven Lern-, Erfahrungs- und Vereinbarungsprozess» konzeptualisiert. Im Hinblick auf dieses Programm folgert sie jedoch, dass sich - trotz des Aufbaus von organisationsübergreifenden Steuerungsstrukturen - das Potential 
zur Entfaltung von Kreativität und inter-organisationalem Lernen nicht entwickeln konnte. Trotz kooperativer und politikfelderübergreifender Steuerungsarrangements bleibe innovative Politikgestaltung, wie sie mit einem Programm wie der «Sozialen Stadt» prinzipiell möglich ist, oftmals das Ergebnis von günstigen und zufälligen Gelegenheiten. Entsprechend folgert HAFNER, dass es einer systematischen Organisation von Lernprozessen bedürfe, um die selbstreflexive Umsteuerung in Gang zu bringen und dauerhaft zu etablieren.

OLIVer Ibert thematisiert in seinem Beitrag multiple Rationalitäten und konzipiert das «Auseinanderfallen» von Akteuren nicht als ein primär ontologisches Problem, sondern als eine erkenntnislogische Strategie. In seinem Beitrag nimmt er Bezug auf die innovationsorientierte Planung und diskutiert zwei Fallstudien aus dem Kontext der IBA Emscher Park im Lichte von Governanz-Verfahren. Er argumentiert, dass sich unter Bedingungen grosser externer Unsicherheit und geringer Umweltkontrolle die Strategiefähigkeit von nur einem kohärenten Akteur nicht aufrecht erhalten lässt. Damit ist Planung als Entscheidungsfindung mit postuliertem erhöhtem Rationalitätsanspruch genuin herausgefordert. IBERT schlägt vor, das «planende Subjekt» aus der modernistischen Planungstheorie analytisch in verschiedene Rollen - Ingenieur, Manager, Kooperationspartner und Moderator - aufzuteilen und jeweils als Bezugspunkt für die Planung zu definieren. An empirischen Beispielen zur «innovationsorientierten Planung» werden die jeweilig zugrunde liegenden Rationalitätskonzepte und die Art, wie in diesen Rollen Entscheidungssequenzen organisiert sind, kontrastiert. Anschliessend werden die instrumentellen Begrenzungen und Potentiale von mit multiplen Rationalitäten operierenden Planungsstrategien - also die Rationalität irrationalen Entscheidens - ausgelotet und diskutiert.

In seinem Beitrag stellt BenedikT Korf die Frage der Legitimität partizipativer Entscheidungsprozesse in den Mittelpunkt. Hiermit öffnet er den Blick auf die Voraussetzungen von Partizipation, welche ihrerseits auf das Spannungsfeld von Legitimitäten, Macht- und Ressourcenkonstellationen in unterschiedlichen Sinnsystemen hinweist. Er geht von der These aus, wonach Partizipation einen «Ausnahmezustand» beschreibt. Hiermit nimmt er Bezug auf Partizipationsformen, die ausserhalb der «normalen», etablierten Institutionen stattfinden. Zudem spricht KorF das oben erwähnte Paradox von alternativen Formen der Entscheidungsfindung an. Laut Korf ist im Ausnahmezustand eine Ineffektivität des Gesetzes wirksam - eine Wirkung ohne Wirkkraft.

Ausgehend von veränderten Problemstellungen in der aktuellen Raumentwicklung konzipiert der Artikel von Joris VAN Wezemael \& MatThIAs LoePfE plane- rische Lösungsversuche als komplexe Prozesse der Entscheidungsfindung. Hierbei wird der Umgang mit Unsicherheit als Ausgangspunkt der Überlegungen gewählt. Durch Aufzeigen dreier aktueller, kontextuell deutlich unterschiedlicher Planungsprojekte aus der Schweiz werden deren bestimmende Momente identifiziert und als «Kipp-Punkte» konzipiert. Vor dem Hintergrund der laufenden Revision des Raumplanungsgesetzes folgert der Beitrag, dass Adaptionsbemühungen je nach gesetzlichen Bedingungen behindert oder unterstiutzt werden.

Das vorliegende Themenheft verdeutlicht das theoretische und methodologische Spektrum der Auseinandersetzung mit Meinungsbildung und Entscheidungsfindung innerhalb der Geographie. Der Arbeitskreis Geographie und Prozesse der Entscheidungsfindung will eine Plattform bieten, um diese Ansätze, Analysen und Konzepte vorzustellen und zu diskutieren. Die Auseinandersetzung mit entscheidungsbezogener Komplexität und Strategien der Komplexitätsreduktion soll dabei zur Stärkung der analytischen Fähigkeiten von Geographen in den Bereichen kollektiver und individueller Entscheidungsfindung beitragen. Eine vertiefte Auseinandersetzung mit den jeweiligen Mechanismen und Hintergründen von Entscheidungsfindungsprozessen in der geographischen Forschung bietet die Basis dafür, dass die Absolvent/innen eines Geographiestudiums längerfristig als Entscheidungsträger oder Berater in komplexen, herausfordernden Bereichen tätig sein und das Bild einer modernen, reflektiven und lösungsorientierten Geographie verbreiten können.

\section{Literatur}

Iraq Body Count Project (IBC, 2008): Iraq Body Count. - http://www.iraqbodycount.org/ 04.04.2009.

National Climatic Data Center (NCDC, 2005): Hurricane Katrina. A climatological perspective. Preliminary Report. Technical Report 2005-01. - http:/www. ncdc.noaa.gov/oa/reports/tech-report-200501z.pdf 04.04.2009.

Van Wezemael, J. \& M. ZaUgG Stern (2006): Gründung eines Arbeitskreises. - In: Geographische Rundschau 58, 3: 64-65.

Prof. Dr. Joris E. Van Wezemael, Universität Freiburg, Departement Geowissenschaften, Geographie, Chemin du Musée 4, CH-1700 Freiburg, Schweiz.

e-mail: vanwezemael@unifr.ch

Dr. Marc Zaugg Sterm, Regionalplanung Zürich und Umgebung RZU, Seefeldstrasse 329, CH-8008 Zürich, Schweiz.

e-mail: zaugg@rzu.ch 